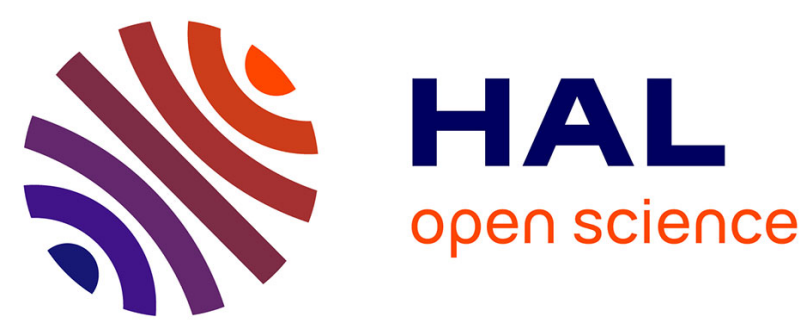

\title{
An Unknown Input Switched Functional Interval Observer for Vehicle Lateral Velocity Estimation
}

\author{
Sara Ifqir, Dalil Ichalal, Naima Ait Oufroukh, Saïd Mammar
}

\section{To cite this version:}

Sara Ifqir, Dalil Ichalal, Naima Ait Oufroukh, Saïd Mammar. An Unknown Input Switched Functional Interval Observer for Vehicle Lateral Velocity Estimation. 58th IEEE Conference on Decision and Control (CDC 2019), Dec 2019, Nice, France. pp.6965-6970, 10.1109/CDC40024.2019.9029497 . hal02444761

\section{HAL Id: hal-02444761 \\ https://hal.science/hal-02444761}

Submitted on 27 Jan 2020

HAL is a multi-disciplinary open access archive for the deposit and dissemination of scientific research documents, whether they are published or not. The documents may come from teaching and research institutions in France or abroad, or from public or private research centers.
L'archive ouverte pluridisciplinaire HAL, est destinée au dépôt et à la diffusion de documents scientifiques de niveau recherche, publiés ou non, émanant des établissements d'enseignement et de recherche français ou étrangers, des laboratoires publics ou privés. 


\title{
An Unknown Input Switched Functional Interval Observer for Vehicle Lateral Velocity Estimation
}

\author{
Sara Ifqir ${ }^{1}$, Dalil Ichalal ${ }^{1}$, Naima Ait-Oufroukh ${ }^{1}$ and Saïd Mammar ${ }^{1}$
}

\begin{abstract}
Functional interval observer of dynamical switched systems provides significant advantages in practical applications. In view of the enlarged order of interval observers, applying interval functional observers can result in lower computational costs and more practicability in some applications such as output feedback control and fault diagnosis of these systems. In this paper, an unknown input functional state interval observer design for a class of switched uncertain systems is investigated. Necessary and sufficient conditions for observer existence are derived. Based on Input to State Stability (ISS) principle and Lyapunov theory, the stability and positivity conditions for the estimation errors are expressed in terms of Linear Matrix Inequalities. A design procedure algorithm of the state observer is given. Finally, the proposed estimation methodology is applied to vehicle lateral velocity estimation problem. Simulation results obtained, confirm the good accuracy and robustness of the proposed state estimation concept.
\end{abstract}

\section{INTRODUCTION}

Recent years have witnessed an increasing interest in investigating state estimation of systems subject to Unknown Inputs (UIs) and modeling uncertainties using intervalobserver-based approaches [6], [5]. This issue, often leading to the so called Unknown Input Interval Observers (UIIOs), is of great concern when dealing with systems with bounded uncertainties. In [7], a robust fault detection methodology using UIIO based on zonotopic set representation for discrete-time linear system is introduced. In [6], an interval observer is built in a new coordinates basis in which the state dynamics is only partially affected by the UIs. In [13], authors deal with the problem of state interval estimation and unknown inputs decoupling for a class of uncertain linear time-invariant system. A robust state estimation problem for a class of switched uncertain linear systems with UIs is investigated in [8], [10]. Furthermore, authors in [9] propose an algorithm to jointly estimate the state and unknown input vector.

It is worth noting, that the interval observers have an enlarged dimension ( $2 n$ variables to estimate the upper and lower bounds of $n$ states), and their implementation requires high degrees of complexity and computational load, especially when dealing with high order complex systems. Thus, the design of functional interval observers [17] that reconstruct directly an upper and a lower bounds of a linear function of the state is of a great importance. In [12] and [2] reduced interval observers have been proposed for linear time-variant

${ }^{1}$ All authors are with IBISC Laboratory, Univ Evry, ParisSaclay university, Evry, 91020, France. E-mail: \{sara.ifqir, dalil.ichalal, naima.aitoufroukh, said.mammar\} duniv-evry.fr and linear time-delay systems. As compared to these works, functional interval observers are flexible and more general. Obviously, functional interval observer is a general form of interval observer because when the linear functions are chosen as the individual unknown states then the problem of functional estimation amounts to the problem of reduced state estimation as in [12], [2]. This feature is particularly advantageous in some applications, such as state feedback control and fault diagnosis. The functional interval observer design problem is addressed in the present paper for the first time, as our main contribution.

Motivated by the above shortcomings, we propose a constructive methodology to design unknown input functional interval observer for switched linear systems with interval uncertainties. Sufficient conditions for the stability and cooperativity of the proposed observer are achieved using the switched ISS-Lyapunov approach with average dwell time switching, and are expressed in terms of Linear Matrix Inequalities (LMIs). An application in the field of vehicle state estimation which has not been treated so far is used to illustrate the design procedure. Validation using real data gathered from a prototype equipped vehicle demonstrates the relevance and efficiency of the proposed interval observer. This paper is organized as follows: System description together with some useful preliminaries are given in Section 2. Section 3 states the main results. First, a new unknown input switched functional interval observer structure is presented and the existence conditions are derived. Finally, design procedure algorithm is included. Simulation result of the application of the proposed interval observer to the problem of vehicle lateral velocity estimation is given in Section 4. Conclusion and future line are detailed in Section 5.

Notation. $\mathbb{R}^{n}$ is the $\mathrm{n}$-dimensional real vector space. The euclidean norm of $x \in \mathbb{R}^{n}$ is denoted by $\|x\| . \underline{x}$ and $\bar{x}$ denote left and right endpoints of an interval $[x]$ such as $[x]=[\underline{x}, \bar{x}]$. Let a vector $x \in \mathbb{R}^{n}$ or a matrix $A \in \mathbb{R}^{n \times n}$, one denotes $x^{+}=\max \{0, x\}, x^{-}=x^{+}-x$ or $A^{+}=\max \{0, A\}$, $A^{-}=A^{+}-A . A>0$ (resp. $A<0$ ) denotes a matrix with positive (resp. negative) components and $A \succ 0$ (resp. $A \prec 0$ ) means that the matrix is positive (resp. negative) definite. $A^{T}$ and $A^{\dagger}=\left(A^{T} A\right)^{-1} A^{T}$ stand to transpose and pseudo-inverse of matrix $A$, respectively. In the sequel, $\lambda_{M}(A)$ (resp. $\left.\lambda_{m}(A)\right)$ denotes the biggest (resp. smallest) eigenvalue of a symmetric matrix $A$. II is identity matrix of appropriate dimension. A real matrix $A$ is called Metzler matrix if all its elements outside the main diagonal are nonnegative. In the rest of the paper, all inequalities must be understood element-wise. 


\section{SYSTEM DESCRIPTION AND PRELIMINARIES}

Let us consider the following uncertain switched dynamical model with $p$ outputs, $m$ inputs and unpredictable unknown input signal $d(t) \in \mathbb{R}^{d}$ :

$$
\left\{\begin{array}{l}
\dot{x}(t)=\left(A_{\sigma(t)}+\Delta A_{\sigma(t)}\right) x(t)+\left(B_{\sigma(t)}+\Delta B_{\sigma(t)}\right) u(t) \\
+E_{\sigma(t)} d(t) \\
y(t)=C x(t)
\end{array},\right.
$$

where $x(t) \in \mathbb{R}^{n}, u(t) \in \mathbb{R}^{m}$ and $y(t) \in \mathbb{R}^{p}$ are the state, input and output vectors, respectively. $\sigma(t): \mathbb{R}^{+} \rightarrow \mathcal{I}=$ $\{1,2, \ldots, N\}$ is a known switching signal, which depends on time $t$, where $N$ denotes the number of subsystems. $A_{\sigma(t)} \in \mathbb{R}^{n \times n}, B_{\sigma(t)} \in \mathbb{R}^{n \times m}, E_{\sigma(t)} \in \mathbb{R}^{n \times d}$ and $C \in$ $\mathbb{R}^{p \times n}$ are known real constant matrices for a fixed $\sigma(t)$. $\Delta A_{\sigma(t)} \in \mathbb{R}^{n \times n}$ and $\Delta B_{\sigma(t)} \in \mathbb{R}^{n \times m}$ are unknown but bounded deviations from the nominal values $A_{\sigma(t)}$ and $B_{\sigma(t)}$, respectively. In this paper, the matrices $\Delta A_{\sigma(t)}$ and $\Delta B_{\sigma(t)}$ are assumed to be bounded and lie into a known interval such that $\forall \sigma(t)$ :

$$
\underline{\Delta A}_{\sigma(t)} \leq \Delta A_{\sigma(t)} \leq \overline{\Delta A}_{\sigma(t)}, \quad \underline{B}_{\sigma(t)} \leq \Delta B_{\sigma(t)} \leq \overline{\Delta B}_{\sigma(t)} .
$$

The uncertain initial condition at the instant $t_{0}, x\left(t_{0}\right)$, is bounded by two known bounds as follows

$$
\underline{x}\left(t_{0}\right) \leq x\left(t_{0}\right) \leq \bar{x}\left(t_{0}\right) .
$$

Furthermore, the control input $u(t)$ in system (1) is subjected to the following constraints:

$$
\underline{u}(t) \leq u(t) \leq \bar{u}(t),
$$

where $\underline{u}(t), \bar{u}(t)$ are known functions.

Let us define a functional state equation described by

$$
z(t)=L_{\sigma(t)} x(t)
$$

where $z(t) \in \mathbb{R}^{r}$ is the vector to be estimated and $L_{\sigma(t)} \in$ $\mathbb{R}^{r \times n}$ is a non-negative known matrix whose pseudo-inverse is written as $L_{\sigma(t)}^{\dagger}=D_{\sigma(t)} L_{\sigma(t)}^{T} \geq 0$ for some positive diagonal matrix $D_{\sigma(t)}$ [1]. Without loss of generality, we assume that: $\operatorname{rank}(C)=p$ and $\operatorname{rank}\left(L_{\sigma(t)}\right)=r, \forall \sigma(t) \in \mathcal{I}$. Since the switched system (1) is uncertain, an exact evaluation of the linear function (5) at each time instant is difficult to obtain. However, it is possible to evaluate all admissible values for $z(t)$ based on (2), (3) and (4). Consider $\bar{z}(t)$ and $\underline{z}(t)$ being the upper and lower estimates of $z(t)$; the obtaining of these two trajectories such that

$$
\underline{z}(t) \leq z(t) \leq \bar{z}(t), \forall t \geq t_{0},
$$

is achieved by using a switched interval functional observer of order $2 r(r \leq n)$. The following useful lemmas are firstly given for further observer design.

Lemma 1. [15] A matrix $A$ is Metzler if and only if there exist $\eta \in \mathbb{R}_{+}$such that $A+\eta \mathcal{I}_{n} \geq 0$.

Definition 1. [11] If $A_{\sigma(t)}$ is Metzler, then the solution of the switched system:

$$
\dot{x}(t)=A_{\sigma(t)} x(t)+\delta_{\sigma(t)}(t),
$$

is non-negative for any $\delta_{\sigma(t)}(t) \geq 0$ and $x\left(t_{0}\right) \geq 0, t \geq t_{0}$. Lemma 2. [3] Let the vector $x(t) \in \mathbb{R}^{n}$ be a variable vector with given bounds $\bar{x}(t), \underline{x}(t) \in \mathbb{R}^{n}$ such that $\underline{x}(t) \leq x(t) \leq$ $\bar{x}(t)$.

1) If $A \in \mathbb{R}^{n \times n}$ is a constant matrix, then

$$
A^{+} \underline{x}(t)-A^{-} \bar{x}(t) \leq A x(t) \leq A^{+} \bar{x}(t)-A^{-} \underline{x}(t) .
$$

2) If $A \in \mathbb{R}^{n \times n}$ is a variable matrix such that $\underline{A} \leq A \leq \bar{A}$ for some $\underline{A}, \bar{A} \in \mathbb{R}^{n \times n}$, then

$$
\begin{aligned}
& \underline{A}^{+} \underline{x}^{+}(t)-\bar{A}^{+} \underline{x}^{-}(t)-\underline{A}^{-} \bar{x}^{+}(t)+\bar{A}^{-} \bar{x}^{-}(t) \leq A x(t) \\
& \leq \bar{A}^{+} \bar{x}^{+}(t)-\bar{A} \underline{x}^{+}(t)-\underline{A}^{+} \bar{x}^{-}(t)+\underline{A}^{-} \underline{x}^{-}(t)
\end{aligned}
$$

Lemma 3. [9] Consider the switched system (7), and let $\varepsilon>$ 0 . Suppose that there exist smooth functions $V_{\sigma(t)}: \mathbb{R}^{n} \rightarrow \mathbb{R}$, such that $V_{\sigma(t)}(x(t))=x^{T}(t) Q_{\sigma(t)} x(t), Q_{\sigma(t)}=Q_{\sigma(t)}^{T} \succ 0$; and constants $\gamma, \alpha_{2}>\alpha_{1}>0$ such that for each $\forall \sigma(t)=$ $i \in \mathcal{I}$, the following conditions hold:

$$
\begin{gathered}
\alpha_{1}\|x(t)\|^{2} \leq V_{\sigma(t)}(x(t)) \leq \alpha_{2}\|x(t)\|^{2}, \\
\dot{V}_{\sigma(t)}(x(t))<-\varepsilon V_{\sigma(t)}(x(t))+\gamma\left\|\delta_{\sigma(t)}(t)\right\|,
\end{gathered}
$$

then, the system (7) is Input-to-State Stable with respect to the additive term $\delta_{\sigma(t)}(t)$ for any switching signal with Average Dwell Time

$$
\tau_{a} \geq \tau_{a}^{*}=\frac{\ln (\mu)}{\varepsilon},
$$

where $\mu=\frac{\alpha_{2}}{\alpha_{1}}$ with $\alpha_{1}=\min _{i \in \mathcal{I}} \lambda_{m}\left(Q_{i}\right)$ and $\alpha_{2}=\max _{i \in \mathcal{I}} \lambda_{M}\left(Q_{i}\right)$.

\section{MAIN RESULT}

Let us consider the following unknown input interval observer structure for system (1) to estimate the upper and lower bounds of $z(t)$ as given in (5)

$$
\left\{\begin{array}{l}
\dot{\bar{\xi}}(t)=N_{\sigma(t)} \bar{\xi}(t)+H_{\sigma(t)} y(t)+G_{\sigma(t)} u(t) \\
+X_{\sigma(t)}^{+} \bar{\delta}_{\sigma(t)}(t)-X_{\sigma(t)}^{-} \underline{\delta}_{\sigma(t)}(t) \\
\bar{z}(t)=\bar{\xi}(t)+M_{\sigma(t)} y(t) \\
\bar{z}\left(t_{0}\right)=L_{\sigma(t)}^{+} \bar{x}\left(t_{0}\right)-L_{\sigma(t)}^{-} \underline{x}\left(t_{0}\right) \\
\dot{\xi}(t)=N_{\sigma(t)} \underline{\xi}(t)+H_{\sigma(t)} y(t)+G_{\sigma(t)} u(t) \\
\quad+X_{\sigma(t)}^{+} \underline{\delta}_{\sigma(t)}(t)-X_{\sigma(t)}^{-} \bar{\delta}_{\sigma(t)}(t) \\
\underline{z}(t)=\underline{\xi}(t)+M_{\sigma(t)} y(t) \\
\underline{z}\left(t_{0}\right)=L_{\sigma(t)}^{+} \underline{x}\left(t_{0}\right)-L_{\sigma(t)}^{-} \bar{x}\left(t_{0}\right)
\end{array}\right.
$$

where $X_{\sigma(t)} \in \mathbb{R}^{r \times n}, N_{\sigma(t)} \in \mathbb{R}^{r \times r}, H_{\sigma(t)} \in \mathbb{R}^{r \times p}, G_{\sigma(t)} \in$ $\mathbb{R}^{r \times m}$ and $M_{\sigma(t)} \in \mathbb{R}^{r \times p}$ are interval functional observer parameters to be determined. $\bar{\delta}_{\sigma(t)}(t)$ and $\underline{\delta}_{\sigma(t)}(t)$ given below are defined using (2), (4), (6) and Lemma 2 such that $\underline{\delta}_{\sigma(t)}(t) \leq \delta_{\sigma(t)}(t) \leq \bar{\delta}_{\sigma(t)}(t)$.

$$
\begin{aligned}
& \bar{\delta}_{\sigma(t)}(t)=\overline{\Delta \mathcal{A}}_{\sigma(t)}^{+} \bar{z}^{+}-\Delta \mathcal{A}_{\sigma(t)}^{+} \bar{z}^{-}-\overline{\Delta \mathcal{A}}_{\sigma(t)}^{-} \underline{z}^{+}+\Delta \mathcal{A}_{\sigma(t)}^{-} \underline{z}^{-} \\
& +\overline{\Delta B}_{\sigma(t)}^{+} \bar{u}^{+}-\underline{\Delta B}{ }_{\sigma(t)}^{+} \bar{u}^{-}-\overline{\Delta B}_{\sigma(t)} \underline{u}^{+}+\underline{B}_{\sigma(t)}^{-} \underline{u}^{-} \\
& \underline{\delta}_{\sigma(t)}(t)=\Delta \mathcal{A}_{\sigma(t)}^{+} \underline{z}^{+}-\overline{\Delta \mathcal{A}}_{\sigma(t)}^{+} \underline{z}^{-}-\Delta \mathcal{A}_{\sigma(t)}^{-} \bar{z}^{+}+\overline{\Delta \mathcal{A}}_{\sigma(t)}^{-} \bar{z}^{-} \\
& +\underline{B}_{\sigma(t)}^{+} \underline{u}^{+}-\overline{\Delta B}_{\sigma(t)}^{+} \underline{u}^{-}-\underline{B}
\end{aligned}
$$

where $\Delta \mathcal{A}_{\sigma(t)}=\Delta A_{\sigma(t)} L_{\sigma(t)}^{\dagger}$. Let $\bar{e}(t)=\bar{z}(t)-z(t)$ and $\underline{e}(t)=z(t)-\underline{z}(t)$ be the upper and lower functional 
estimation errors, using (13), $\bar{e}(t)$ and $\underline{e}(t)$ are rewritten as follows:

$$
\begin{aligned}
& \bar{e}(t)=\bar{\xi}(t)-\left(L_{\sigma(t)}-M_{\sigma(t)} C\right) x(t), \\
& \underline{e}(t)=\left(L_{\sigma(t)}-M_{\sigma(t)} C\right) x(t)-\underline{\xi}(t) .
\end{aligned}
$$

Define

$$
X_{\sigma(t)}=L_{\sigma(t)}-M_{\sigma(t)} C
$$

Then, by taking the time derivative of (15), the upper and lower errors dynamics are obtained as follows

$$
\begin{aligned}
& \dot{\bar{e}}(t)=N_{\sigma(t)} \bar{e}(t)+\left(G_{\sigma(t)}-X_{\sigma(t)} B_{\sigma(t)}\right) u(t)+ \\
& \left(N_{\sigma(t)} X_{\sigma(t)}+H_{\sigma(t)} C-X_{\sigma(t)} A_{\sigma(t)}\right) x(t)+ \\
& X_{\sigma(t)}^{+} \delta_{\sigma(t)}(t)-X_{\sigma(t)}^{-} \underline{\delta}_{\sigma(t)}(t)-X_{\sigma(t)} \delta_{\sigma(t)}(t) \\
& -X_{\sigma(t)} E_{\sigma(t)} d(t), \\
& \underline{\dot{e}}(t)=N_{\sigma(t)} \underline{e}(t)-\left(G_{\sigma(t)}-X_{\sigma(t)} B_{\sigma(t)}\right) u(t)- \\
& \left(N_{\sigma(t)} X_{\sigma(t)}+H_{\sigma(t)} C-X_{\sigma(t)} A_{\sigma(t)}\right) x(t)+ \\
& X_{\sigma(t)} \delta_{\sigma(t)}-X_{\sigma(t)}^{+} \underline{\delta}_{\sigma(t)}(t)+X_{\sigma(t)}^{-} \bar{\delta}_{\sigma(t)}(t) \\
& +X_{\sigma(t)} E_{\sigma(t)} d(t) .
\end{aligned}
$$

From (17), $\bar{z}(t)$ and $\underline{z}(t)$ are the upper and lower bounds of $z(t)$ if the following conditions hold.

Proposition 1. The linear function (5) and the solutions of (13) satisfy:

$$
\underline{z}(t) \leq z(t) \leq \bar{z}(t),
$$

if $\forall \sigma(t)$

$$
\begin{gathered}
N_{\sigma(t)} \text { is Metzler; } \\
N_{\sigma(t)} X_{\sigma(t)}+H_{\sigma(t)} C-X_{\sigma(t)} A_{\sigma(t)}=0 ; \\
G_{\sigma(t)}-X_{\sigma(t)} B_{\sigma(t)}=0 ; \\
X_{\sigma(t)} E_{\sigma(t)}=0 .
\end{gathered}
$$

Proof. If conditions (19b-19d) are satisfied then (17) is reduced to

$$
\left\{\begin{array}{l}
\dot{\bar{e}}(t)=N_{\sigma(t)} \bar{e}(t)+\bar{\Delta}_{\sigma(t)}(t) \\
\underline{\dot{e}}(t)=N_{\sigma(t)} \underline{e}(t)+\underline{\Delta}_{\sigma(t)}(t)
\end{array},\right.
$$

where

$$
\begin{aligned}
& \bar{\Delta}_{\sigma(t)}(t)=X_{\sigma(t)}^{+} \bar{\delta}_{\sigma(t)}(t)-X_{\sigma(t)}^{-} \underline{\delta}_{\sigma(t)}(t)-X_{\sigma(t)} \delta_{\sigma(t)}(t), \\
& \underline{\Delta}_{\sigma(t)}(t)=X_{\sigma(t)} \delta_{\sigma(t)}(t)-X_{\sigma(t)}^{+} \underline{\delta}_{\sigma(t)}(t)+X_{\sigma(t)}^{-} \bar{\delta}_{\sigma(t)}(t) .
\end{aligned}
$$

Since $\bar{\Delta}_{\sigma(t)}(t) \geq 0$ and $\underline{\Delta}_{\sigma(t)}(t) \geq 0$ by construction $\forall t \geq$ $t_{0}$, then, $\bar{e}(t) \geq 0$, i.e. $\bar{z}(t) \geq z(t)$ and $\underline{e}(t) \geq 0$, i.e. $z(t) \geq$ $\underline{z}(t)$ provided that $N_{\sigma(t)}$ is Metzler $\forall \sigma(t)$ and $\bar{e}\left(t_{0}\right) \geq 0$ and $\underline{e}\left(t_{0}\right) \geq 0, \forall t \geq t_{0}$ according to Definition 1 .

The Proposition 1 does not claim that variables $\underline{z}(t)$ and $\bar{z}(t)$ are bounded, it establishes only the order relations (18). To ensure the boundedness of solutions for the system (13), we introduce the interval functional estimation error $e(t)$, which is defined to be the difference between the upper and lower estimates $\bar{z}(t)$ and $\underline{z}(t)$ as follows

$$
e(t)=\bar{z}(t)-\underline{z}(t),
$$

then the error dynamics is given by

$$
\dot{e}(t)=N_{\sigma(t)} e(t)+\Delta_{\sigma(t)}(t),
$$

where

$$
\Delta_{\sigma(t)}(t)=\bar{\Delta}_{\sigma(t)}(t)-\underline{\Delta}_{\sigma(t)}(t) .
$$

Proposition 2. As long as the plant's input $u(t)$ and state $x(t)$ remain bounded, and $N_{\sigma(t)}$ is chosen to be Hurwitz $\forall \sigma(t)$, then the interval estimation error (24) is robust to the additive uncertainties $\Delta_{\sigma(t)}$ in the ISS sense [16].

Proof. Let us choose a a Switched ISS-Lyapunov function candidate [9] as follows:

$$
V_{\sigma(t)}(e(t))=e^{T}(t) Q_{\sigma(t)} e(t),
$$

where $Q_{\sigma(t)}$ is a diagonal matrix such that $Q_{\sigma(t)}=Q_{\sigma(t)}^{T} \geq$ $0, \forall \sigma(t)$. Taking the time derivative of $V_{\sigma(t)}(e(t))$ along the trajectory of the interval functional estimation error (24) in each mode $i$ yields

$$
\begin{aligned}
& \dot{V}_{i}(e(t))=e^{T}(t)\left(N_{i}^{T} Q_{i}+Q_{i} N_{i}\right) e(t)+ \\
& \Delta_{i}^{T}(t) Q_{i} e(t)+e^{T}(t) Q_{i} \Delta_{i}(t) .
\end{aligned}
$$

By adding and subtracting the terms $\varepsilon e^{T}(t) Q_{i} e(t)$ and $-\gamma \Delta_{i}^{T}(t) \Delta_{i}(t),(27)$ becomes

$$
\begin{aligned}
& \dot{V}_{i}(e(t))=\left[\begin{array}{c}
e^{T}(t) \\
\Delta_{i}^{T}(t)
\end{array}\right] \Gamma_{i}\left[\begin{array}{c}
e(t) \\
\Delta_{i}(t)
\end{array}\right], \\
& -\varepsilon V_{i}(e(t))+\gamma \Delta_{i}^{T}(t) \Delta_{i}(t)
\end{aligned}
$$

where

$$
\Gamma_{i}=\left[\begin{array}{cc}
N_{i}^{T} Q_{i}+Q_{i} N_{i}+\varepsilon Q_{i} & Q_{i} \\
Q_{i} & -\gamma \mathbb{I}
\end{array}\right] .
$$

Then, if

$$
\Gamma_{i} \prec 0,
$$

it follows that

$$
\dot{V}_{i}(e(t))<-\varepsilon V_{i}(e(t))+\gamma \Delta_{i}^{T}(t) \Delta_{i}(t),
$$

by integrating the inequality (30) over the interval $\left[t_{k}, t\right)$, one obtains

$V_{i}(e(t))<\mathrm{e}^{-\varepsilon\left(t-t_{k}\right)} V_{i}\left(e\left(t_{k}\right)\right)+\gamma \int_{t_{k}}^{t} \mathrm{e}^{-\varepsilon\left(\left(t-t_{k}\right)-s\right)}\left\|\Delta_{i}(s)\right\|_{2}^{2} \mathrm{~d} s$.

Using (10), we obtain

$$
\|e(t)\|_{2}^{2}<\frac{1}{\alpha_{1}}\left(\mathrm{e}^{-\varepsilon\left(t-t_{k}\right)} V_{i}\left(e\left(t_{k}\right)\right)+\frac{\gamma}{\varepsilon}\left\|\Delta_{i}(t)\right\|_{\infty}^{2}\right),
$$

then

$$
\|e(t)\|_{2}<\frac{1}{\sqrt{\alpha_{1}}}\left(\mathrm{e}^{-\varepsilon\left(t-t_{k}\right)} V_{i}\left(t_{k}\right)+\frac{\gamma}{\varepsilon}\left\|\Delta_{i}(t)\right\|_{\infty}^{2}\right)^{\frac{1}{2}} .
$$

Therefore, it can be concluded that if $\left\|\Delta_{i}(t)\right\|_{\infty}=0$, then $\|e(t)\|_{2} \rightarrow 0$ when $t \rightarrow \infty$. Moreover, in the presence of uncertainties, the interval error $\|e(t)\|_{2}$ is upper bounded by $\sqrt{\frac{\gamma}{\alpha_{1} \varepsilon}} \max _{i \in \mathcal{I}}\left\|\Delta_{i}(t)\right\|_{\infty}$. This statement completes the proof according to the definition of ISS principle.

Remark 1. It is noted that, if the deviations from the nominal values are absent (i.e., $\Delta A_{\sigma(t)}=0$ and $\Delta B_{\sigma(t)}=$ 0 ), the functional interval observer (13) becomes a so-called globally convergent functional interval observer meaning that $\lim _{t \rightarrow \infty} e(t)=0$, i.e. $\underline{z}(t) \rightarrow z(t) \leftarrow \bar{z}(t)$ for any initial conditions $\underline{z}\left(t_{0}\right) \leq z\left(t_{0}\right) \leq \bar{z}\left(t_{0}\right)$. However, in the presence of bounded additive uncertainties (i.e., $\Delta A_{\sigma(t)} \neq 0$ and $\Delta B_{\sigma(t)} \neq 0$ ), the switched interval observer (13) should generate an upper and lower estimates of the linear function $L_{\sigma(t)} x(t)$ with the interval estimation error satisfying an ISS contraction property with respect to $\Delta_{\sigma(t)}(t)$.

In the sequel, a constructive methodology for solving constraints (19b-19d) with the requirement that $N_{\sigma(t)}$ is Hurwitz 
and Metzler $\forall \sigma(t)$ and the interval functional estimation error (24) is ISS with respect to additive uncertainties $\Delta_{\sigma(t)}(t)$ is proposed.

By substituting $X_{\sigma(t)}$ from (16) into (19b), we obtain

$$
N_{\sigma(t)} L_{\sigma(t)}=L_{\sigma(t)} A_{\sigma(t)}-\left[\begin{array}{ll}
M_{\sigma(t)} & T_{\sigma(t)}
\end{array}\right] \Lambda_{\sigma(t)},
$$
where $T_{\sigma(t)}=H_{\sigma(t)}-N_{\sigma(t)} M_{\sigma(t)}$ and $\Lambda_{\sigma(t)}=\left[\begin{array}{c}C A_{\sigma(t)} \\ C\end{array}\right]$.
Define the following full row-rank switched matrix

$$
\left[\begin{array}{ll}
Q_{\sigma(t)} & W_{\sigma(t)}
\end{array}\right]=\left[\begin{array}{ll}
L_{\sigma(t)}^{\dagger} & \left(\mathbb{I}-L_{\sigma(t)}^{\dagger} L_{\sigma(t)}\right)
\end{array}\right]
$$

multiplying both sides of (34) by (35) leads to

$$
\begin{gathered}
N_{\sigma(t)}=L_{\sigma(t)} A_{\sigma(t)} Q_{\sigma(t)}-\left[\begin{array}{ll}
M_{\sigma(t)} & T_{\sigma(t)}
\end{array}\right] \Lambda_{\sigma(t)} Q_{\sigma(t)}, \\
L_{\sigma(t)} A_{\sigma(t)} Q_{\sigma(t)}=\left[\begin{array}{ll}
M_{\sigma(t)} & T_{\sigma(t)}
\end{array}\right] \Lambda_{\sigma(t)} W_{\sigma(t)} .
\end{gathered}
$$

From the definition of $X_{\sigma(t)}$, condition (19d) is written as

$$
L_{\sigma(t)} E_{\sigma(t)}=M_{\sigma(t)} C E_{\sigma(t)} .
$$

Combining equations (37) and (38) gives

$$
\left[\begin{array}{ll}
M_{\sigma(t)} & T_{\sigma(t)}
\end{array}\right] \Omega_{\sigma(t)}=\Psi_{\sigma(t)},
$$

where $\Omega_{\sigma(t)}=\left[\begin{array}{cc}C A_{\sigma(t)} W_{\sigma(t)} & C E_{\sigma(t)} \\ C W_{\sigma(t)} & 0\end{array}\right]$ and $\Psi_{\sigma(t)}=$ $\left[L_{\sigma(t)} A_{\sigma(t)} Q_{\sigma(t)} \quad L_{\sigma(t)} E_{\sigma(t)}\right]$.

The following lemma provides the necessary and sufficient conditions for the existence of a solution to (39).

Lemma 4. There exists a solution to equation (39) if and only if

$$
\begin{gathered}
\operatorname{rank}\left[\begin{array}{cc}
C_{\sigma(t)} A_{\sigma(t)} & C_{\sigma(t)} E_{\sigma(t)} \\
C_{\sigma(t)} & 0 \\
L_{\sigma(t)} A_{\sigma(t)} & L_{\sigma(t)} E_{\sigma(t)} \\
L_{\sigma(t)} & 0
\end{array}\right]= \\
\operatorname{rank}\left[\begin{array}{cc}
C_{\sigma(t)} A_{\sigma(t)} & C_{\sigma(t)} E_{\sigma(t)} \\
C_{\sigma(t)} & 0 \\
L_{\sigma(t)} & 0
\end{array}\right], \forall \sigma(t) .
\end{gathered}
$$

Proof. The necessary and sufficient condition for the existence of a solution to (39) is

$$
\operatorname{rank}\left[\begin{array}{l}
\Omega_{\sigma(t)} \\
\Psi_{\sigma(t)}
\end{array}\right]=\operatorname{rank}\left(\Omega_{\sigma(t)}\right)
$$

Let $\mathcal{S}_{\sigma(t)}$ be a full-row rank matrix $\forall \sigma(t)$ defined as:

$$
\mathcal{S}_{\sigma(t)}=\left[\begin{array}{ccc}
Q_{\sigma(t)} & W_{\sigma(t)} & 0 \\
0 & 0 & \mathbb{I}
\end{array}\right]
$$

then, we have

$$
\begin{aligned}
& \operatorname{rank}\left[\begin{array}{cc}
C_{\sigma(t)} A_{\sigma(t)} & C_{\sigma(t)} E_{\sigma(t)} \\
C_{\sigma(t)} & 0 \\
L_{\sigma(t)} A_{\sigma(t)} & L_{\sigma(t)} E_{\sigma(t)} \\
L_{\sigma(t)} & 0
\end{array}\right] \\
& \left.=\operatorname{rank}\left[\begin{array}{cc}
C_{\sigma(t)} A_{\sigma(t)} & C_{\sigma(t)} E_{\sigma(t)} \\
C_{\sigma(t)} & 0 \\
L_{\sigma(t)} A_{\sigma(t)} & L_{\sigma(t)} E_{\sigma(t)} \\
L_{\sigma(t)} & 0
\end{array}\right] \mathcal{S}_{\sigma(t)}\right) \\
& =\operatorname{rank}\left[\begin{array}{ccc}
C_{\sigma(t)} A_{\sigma(t)} Q_{\sigma(t)} & C_{\sigma(t)} A_{\sigma(t)} W_{\sigma(t)} & C_{\sigma(t)} E_{\sigma(t)} \\
C_{\sigma(t)} Q_{\sigma(t)} & C_{\sigma(t)} W_{\sigma(t)} & 0 \\
L_{\sigma(t)} A_{\sigma(t)} Q_{\sigma(t)} & L_{\sigma(t)} A_{\sigma(t)} W_{\sigma(t)} & L_{\sigma(t)} E_{\sigma(t)} \\
\mathbb{I} & 0
\end{array}\right] \\
& =\operatorname{rank}\left[\begin{array}{c}
\Omega_{\sigma(t)} \\
\Psi_{\sigma(t)}
\end{array}\right] .
\end{aligned}
$$

On the other hand,

$$
\begin{aligned}
& \operatorname{rank}\left[\begin{array}{cc}
C_{\sigma(t)} A_{\sigma(t)} & C_{\sigma(t)} E_{\sigma(t)} \\
C_{\sigma(t)} & 0 \\
L_{\sigma(t)} & 0
\end{array}\right] \\
& =\operatorname{rank}\left(\left[\begin{array}{cc}
C_{\sigma(t)} A_{\sigma(t)} & C_{\sigma(t)} E_{\sigma(t)} \\
C_{\sigma(t)} & 0 \\
L_{\sigma(t)} & 0
\end{array}\right] \mathcal{S}_{\sigma(t)}\right) \\
& =\operatorname{rank}\left[\begin{array}{ccc}
C_{\sigma(t)} A_{\sigma(t)} Q_{\sigma(t)} & C_{\sigma(t)} A_{\sigma(t)} W_{\sigma(t)} & C_{\sigma(t)} E_{\sigma(t)} \\
C_{\sigma(t)} Q_{\sigma(t)} & C_{\sigma(t)} W_{\sigma(t)} & 0 \\
\mathbb{I} & 0 & 0
\end{array}\right] \\
& =\operatorname{prank}\left(\Omega_{\sigma(t)}\right) .
\end{aligned}
$$

Using (41), (40) is obtained.

Under condition (40), a general solution of equation (39) is given by:

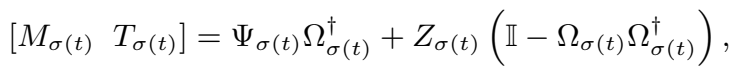

where $Z_{\sigma(t)}$ is a switched arbitrary matrix of appropriate dimensions. By substituting the solution (43) into (36), gives

$$
N_{\sigma(t)}=\Theta_{\sigma(t)}-Z_{\sigma(t)} \Delta_{\sigma(t)},
$$

where $\Theta_{\sigma(t)}=L_{\sigma(t)} A_{\sigma(t)} Q_{\sigma(t)}-\Psi_{\sigma(t)} \Omega_{\sigma(t)}^{\dagger} \Lambda_{\sigma(t)} Q_{\sigma(t)}$ and $\Delta_{\sigma(t)}=\left(\mathbb{I}-\Omega_{\sigma(t)} \Omega_{\sigma(t)}^{\dagger}\right) \Lambda_{\sigma(t)} Q_{\sigma(t)}$.

By substituting (43) into (16), the matrix $X_{\sigma(t)}$ can be reexpressed as

$$
\begin{aligned}
& X_{\sigma(t)}=L_{\sigma(t)}-\Psi_{\sigma(t)} \Omega_{\sigma(t)}^{\dagger}\left[\begin{array}{ll}
C & 0
\end{array}\right]^{T} \\
& +Z_{\sigma(t)}\left(\mathbb{I}-\Omega_{\sigma(t)} \Omega_{\sigma(t)}^{\dagger}\right)\left[\begin{array}{ll}
C & 0
\end{array}\right]^{T} .
\end{aligned}
$$

Now, the design problem of the switched functional interval observer (13) is reduced to finding the matrix $Z_{\sigma(t)}$ such that conditions of Propositions 1 and 2 are fulfilled. The Theorem 1 states the main results.

Theorem 1. Assume that there exist positive diagonal matrix $Q_{i}$ and matrix $Y_{i}$ for given constants $\eta_{\sigma(t)} \geq 0$ and $\varepsilon>$ 0 such that the following convex optimization problem is solvable for all $i \in \mathcal{I}$ :

$$
\min _{Q_{i}, Y_{i}} \gamma
$$

subject to

$$
\begin{gathered}
\alpha_{1} \mathcal{I}_{n} \preceq Q_{i} \preceq \beta \mathcal{I}_{n} \\
{\left[\begin{array}{cc}
\Theta_{i}^{T} Q_{i}-\Delta_{i}^{T} Y_{i}^{T}+Q_{i} \Theta_{i}-Y_{i} \Delta_{i}+\varepsilon Q_{i} & Q_{i} \\
Q_{i} & -\gamma \mathbb{I}
\end{array}\right] \prec 0,} \\
Q_{i} \Theta_{i}-Y_{i} \Delta_{i}+\eta Q_{i} \geq 0,
\end{gathered}
$$

then, the interval functional error (24) is Input-to-State Stable for any switching signal with the average dwell time (12), with $Z_{i}=Q_{i}^{-1} Y_{i}$.

Proof. From Proposition 2, it has been shown that if inequality (29) is satisfied, then the interval functional estimation error (24) is ISS with respect to $\Delta_{\sigma(t)}(t)$ with the ISS gain $\sqrt{\frac{\gamma}{\alpha_{1} \varepsilon}}$. Now, by replacing $N_{i}$ in (29) by (44) and denote $Y_{i}=Q_{i} Z_{i}$, inequality (47) is directly deduced. On the other hand, according to Lemma $1, N_{\sigma(t)}$ is Metzler if

$$
N_{i}+\eta_{i} \mathcal{I}_{n} \geq 0, \forall i \in \mathcal{I}
$$


multiplying in the left side by $Q_{i}$ and using (44) together with the change of coordinates $Y_{i}=Q_{i} Z_{i}$, (48) is obtained.

The convex optimization problem given in Theorem 1 states that the optimal gain $\gamma$ which explicitly bounds the interval estimation error in the steady state is obtained by selecting among all possible solutions $Q_{i}, Y_{i}$ those leading to the smallest value for $\gamma$ and therefore a smallest convergence region.

Overall, the unknown input switched interval functional observer parameters can be calculated based on the following algorithm:

Step i: Solve the optimization problem in Theorem 1 for given $\varepsilon$ and $\eta_{\sigma(t)}$ and obtain $Z_{\sigma(t)}, \forall \sigma(t)=i \in \mathcal{I}$.

Step ii: Calculate matrices $N_{\sigma(t)}$ and $X_{\sigma(t)}$ in accordance with their definitions given in (44) and (45). Use (43) to obtain $M_{\sigma(t)}$ and $T_{\sigma(t)}$.

Step iii Obtain $G_{\sigma(t)}=X_{\sigma(t)} B_{\sigma(t)}$ and $G_{\sigma(t)}=T_{\sigma(t)}+$ $N_{\sigma(t)} M_{\sigma(t)}$.

\section{Application to Vehicle LATERAL VElocity ESTIMATION}

Vehicle lateral velocity estimation has become the most challenging aspect since the introduction of the first on-board active safety systems controlling vehicle stability, such as the Electronic stability control (ESC), also referred to as Electronic Stability Program (ESP). Recently, Vehicle Control Systems (VCS) such as active steering, direct yaw control (DYC), advanced traction control and the aforementioned ESP are used together to improve vehicle handling stability. All of these advanced control systems rely on an accurate knowledge of vehicle dynamic states, and on vehicle lateral velocity monitoring in particular. Therefore, looking for an estimation method with high precision and good real-time is very crucial. In this section, based on a 2-DOF vehicle model and the proposed functional observer, an estimate of the vehicle lateral velocity is proposed and evaluated using experimental data.

\section{A. Vehicle model}

The basis of the proposed lateral velocity estimator is the bicycle vehicle model [14], which describes the lateral and yaw motions of a 2-axle, 1-rigid body ground vehicle. The

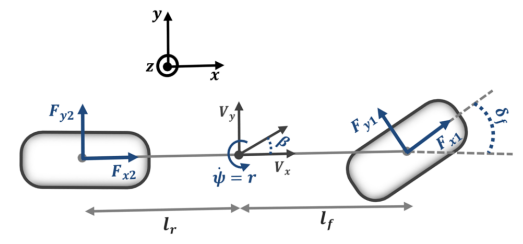

Fig. 1. Bicycle model.

dynamics equations with side-slip angle $\beta$ and yaw rate $\dot{\psi}$ selected as state variables are given as follows:

$\left[\begin{array}{c}\dot{\beta} \\ \ddot{\psi}\end{array}\right]=\left[\begin{array}{cc}-\frac{c_{f}+c_{r}}{m v_{x}} & \frac{c_{r} l_{r}-c_{f} l_{f}}{m v_{x}^{2}}-1 \\ \frac{c_{r} l_{r}-c_{f} l_{f}}{I_{z}} & -\frac{c_{r} l_{r}^{2}+c_{f} l_{f}^{2}}{I_{z} v_{x}}\end{array}\right]\left[\begin{array}{c}\beta \\ \dot{\psi}\end{array}\right]+\left[\begin{array}{c}\frac{c_{f}}{m v_{x}} \\ \frac{c_{f} l_{f}}{I z}\end{array}\right] \delta_{f}+\left[\begin{array}{c}\frac{1}{m v_{x}} \\ \frac{l_{w}}{I_{z}}\end{array}\right] f_{w}$ where $m$ is vehicle mass, $I_{z}$ is yaw inertia moment, $v_{x}$ is longitudinal velocity, $\delta_{f}$ is front wheel steering angle. $l_{f}$ and $l_{r}$ are distances between the centre of gravity and the front and rear axles, respectively. $f_{w}$ is the force due to the effect of the wind gusts that are acting at a distance $l_{w} . c_{f}$ and $c_{r}$ are the front and rear tire cornering stiffness coefficients. The available measurement output is the yaw rate $r$.

The parameters of the vehicle lateral dynamic model (50) are assumed to be certain, except cornering stiffness coefficients, which are the most influential and unpredictable ones. An adaptation of the vehicle model (50) is done by decomposing these parameters as follows:

$$
c_{f}=c_{f_{0}}+\Delta c_{f}, \quad c_{r}=c_{r_{0}}+\Delta c_{r}
$$

where $c_{i 0}, \mathrm{i} \in\{r, f\}$ is a known nominal value, for example, determined on a nominal surface, and $\Delta c_{i} \in\left[\Delta c_{i}^{-}, \Delta c_{i}^{+}\right], \mathrm{i}$ $\in\{r, f\}$ is a time varying, unknown but bounded term. On the other hand, in order to deal with longitudinal velocity variation, we transform the uncertain system (50) into a switched uncertain system where each subsystem operates around a given constant longitudinal velocity value (for example, three subsystems defined for low, average and high longitudinal speed). According to these considerations, and by substituting (51) into (50) yields to a state-space model of the form (1). The switching signal $\sigma(t)$ depends in this case on the measured vehicle longitudinal velocity. Now, to illustrate the design method in this paper, recall that the side slip angle $\beta$ can be related to vehicle lateral velocity $v_{y}$ (under small angle assumptions) as: $v_{y}=\beta v_{x}$, then a switched functional interval observer can be built to estimate the state $z(t)=v_{y}=\left[\begin{array}{ll}v_{x} & 0\end{array}\right] x(t)$ following the design procedure presented in the previous section.

\section{B. Simulation results}

Now, we shall use numerical simulation to verify the effectiveness of the proposed lateral velocity estimation scheme. The real data used in the validation process are based on measurements acquired using a prototype vehicle in a test track located in the city of Versailles-Satory, France. The vehicle is equipped with an inertial unit, an odometer and an absolute optical encoder measuring respectively, the yaw rate, the vehicle longitudinal speed and the steering angle. The effect of wind lateral force acting as unknown input is added in simulation to real acquired data. Effect of the added wind force is incorporated into the measurements using the nonlinear vehicle model [4]. Steering angle, yaw rate, longitudinal velocity and lateral wind force profiles are shown in Figure 2. For the simulation scenario, in order to deal with the time-varying velocity, three subsystems are considered for $v_{x}^{1}=8.5 \mathrm{~m} / \mathrm{s}, v_{x}^{2}=13.55 \mathrm{~m} / \mathrm{s}$ and $v_{x}^{3}=18.05 \mathrm{~m} / \mathrm{s}$. The switching signal illustrated in Figure 3 is defined as:

$$
\sigma(t)=\left\{\begin{array}{lll}
1 & \text { if } & v_{x} \in\left[V_{x}^{0}, V_{x}^{1}[\right. \\
2 & \text { if } & v_{x} \in\left[V_{x}^{1}, V_{x}^{2}[\right. \\
3 & \text { if } & v_{x} \in\left[V_{x}^{2}, V_{x}^{3}\right]
\end{array},\right.
$$

where $v_{x}^{k}=\frac{V_{x}^{k}-V_{x}^{k-1}}{2}$ for $k=1,2,3$. In addition, we assume that the cornering stiffness coefficients are affected by $\pm 30 \%$ 

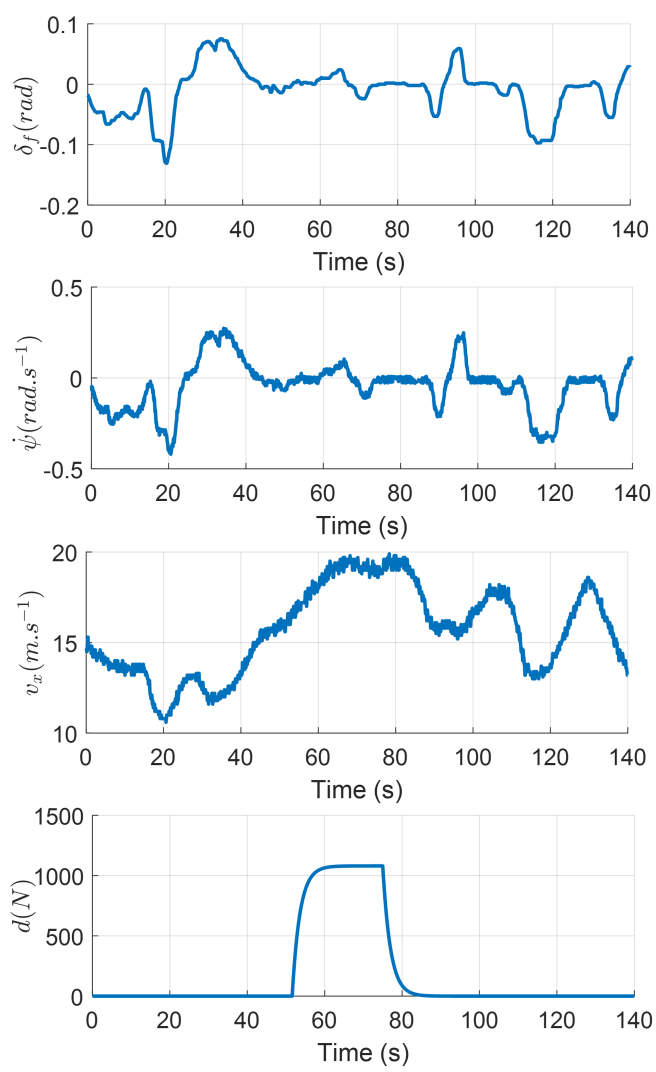

Fig. 2. Steering angle input $\delta_{f}$, yaw rate $\dot{\psi}$, Longitudinal velocity $v_{x}$ and Wind lateral force $f_{w}$.

uncertainty in their nominal values, which represents a realistic estimate of the variation range (from dry asphalt to ice-covered roads). Choosing $\varepsilon=0.2, \eta_{1}=\eta_{2}=\eta_{3}=0.1$

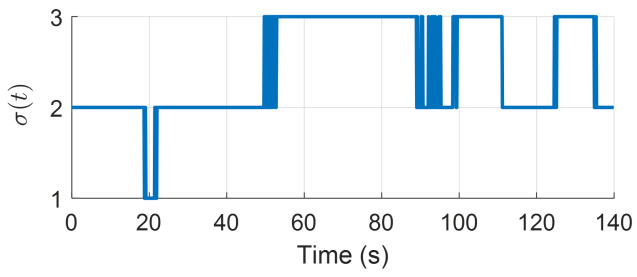

Fig. 3. Switching signal $\sigma(t)$.

and solving LMIs 46-48, we obtain

$$
\begin{aligned}
& Z_{1}=10^{-14}[0.3065-0.0766], Z_{2}=10^{-14}[0.3065-0.0766], \\
& Z_{3}=10^{-14}[0.3065-0.0766], X_{1}=[8.0000-3.8344] \\
& X_{2}=[12.5000-3.8344], X_{3}=[17.5000-3.8344] \\
& N_{1}=-9.9287, N_{2}=-6.3544, N_{3}=-4.5388, P_{1}=0.0168, \\
& P_{2}=0.0173, P_{3}=0.0200, \gamma=0.0023 .
\end{aligned}
$$

The result of the switched functional interval observer is given in Figure 4. The vehicle lateral velocity remains inside the estimated interval $\left[v_{y}^{-}, v_{y}^{+}\right]$which confirms the accuracy and efficiency of the proposed method.

\section{CONCLUSION}

An unknown input functional interval observer has been designed for switched linear systems with bounded uncertainties. A method based on unknown input decoupling, ISS

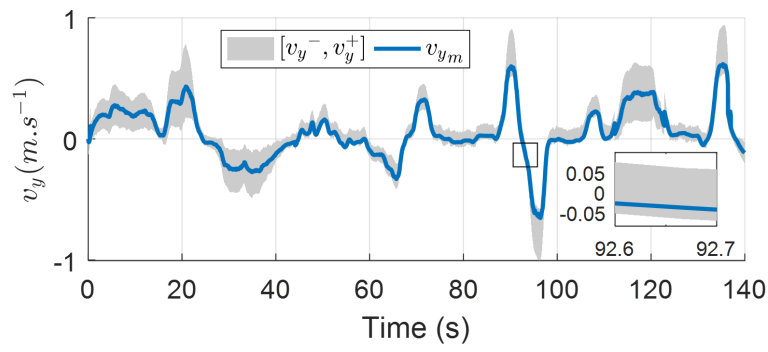

Fig. 4. Vehicle lateral velocity interval estimation.

principle and switched Lyapunov theory has been presented. The existence conditions of the proposed observer have been established. Application to vehicle lateral velocity estimation demonstrates the effectiveness of the proposed design method. Future work will be devoted to extend the presented technique to uncertain switched linear parameter varying systems.

\section{REFERENCES}

[1] Abraham Berman and Robert Plemmons. Nonnegative matrices in the mathematical sciences, volume 9. Siam, 1994.

[2] Denis Efimov, Wilfrid Perruquetti, and Jean-Pierre Richard. On reduced-order interval observers for time-delay systems. In 2013 European Control Conference (ECC), pages 2116-2121. IEEE, 2013.

[3] Denis Efimov, Tarek Raïssi, Stanislav Chebotarev, and Ali Zolghadri. Interval state observer for nonlinear time varying systems. Automatica, 49(1):200-205, 2013

[4] Thomas D Gillespie. Fundamentals of vehicle dynamics. Technical report, SAE Technical Paper, 1992.

[5] David Gucik-Derigny, Tarek Raïssi, and Ali Zolghadri. Interval state and unknown inputs estimation for linear time-invariant systems. IFAC Proceedings Volumes, 47(3):7375-7381, 2014.

[6] David Gucik-Derigny, Tarek Raïssi, and Ali Zolghadri. A note on interval observer design for unknown input estimation. International Journal of Control, 89(1):25-37, 2016.

[7] Pedro Guerra, Vicenc Puig, and Marcin Witczak. Robust fault detection with unknown-input interval observers using zonotopes. IFAC Proceedings Volumes, 41(2):5557-5562, 2008.

[8] Sara Ifqir, Naima Ait-Oufroukh, Dalil Ichalal, and Saïd Mammar Switched unknown inputs interval observer design for vehicle lateral dynamics estimation with wind gusts rejection. 14th International Workshop on Advanced Control and Diagnosis, 2017.

[9] Sara Ifqir, Dalil Ichalal, Naima Ait Oufroukh, and Saïd Mammar. Robust interval observer for switched systems with unknown inputs: application to vehicle dynamics estimation. European Journal of Control, 44:3-14, 2018.

[10] Sara Ifqir, Naima Ait Oufroukh, Dalil Ichalal, and Saïd Mammar. Vehicle lateral dynamics estimation using switched unknown inputs interval observers: Experimental validation. IEEE Annual American Control Conference (ACC), pages 1138-1143, 2018.

[11] Tadeusz Kaczorek. Positive 1d and 2d systems. communications and control engineering, 2002.

[12] Stefan Krebs, Christoph Schnurr, Martin Pfeifer, Jörg Weigold, and Sören Hohmann. Reduced-order hybrid interval observer for verified state estimation of an induction machine. Control Engineering Practice, 57:157-168, 2016

[13] David Gucik-Derigny Nicolas Ellero and David Henry. Unknown input interval observer with $\mathrm{h}$ and d-stability performance. IFACPapersOnLine, 50(1):6251-6258, 2017.

[14] Rajesh Rajamani. Vehicle dynamics and control. Springer Science \& Business Media, 2011.

[15] M Ait Rami, Chun Hung Cheng, and C De Prada. Tight robust interval observers: an LP approach. In Decision and Control, 2008. CDC 2008. 47th IEEE Conference on, pages 2967-2972. IEEE, 2008.

[16] Eduardo D Sontag and Yuan Wang. Notions of input to output stability. Systems \& Control Letters, 38(4-5):235-248, 1999.

[17] Hieu Trinh and Tyrone Fernando. Functional observers for dynamical systems, volume 420. Springer Science \& Business Media, 2011. 